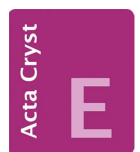

CRYSTALLOGRAPHIC COMMUNICATIONS

ISSN 2056-9890

Received 17 April 2018

Accepted 23 April 2018

Edited by W. T. A. Harrison, University of Aberdeen, Scotland

Keywords: octane-1,8-diaminium 4,4'-azinodibenzoate; crystal structure; ionic pseudopolymer; 4,4'-azinodibenzoic acid.

CCDC reference: 1839024

Supporting information: this article has supporting information at journals.iucr.org/e

\section{Crystal structure of octane-1,8-diaminium 4,4'-(diazene-1,2-diyl)dibenzoate monohydrate}

\author{
Igor Elkin, ${ }^{\mathrm{a}}$ Thierry Maris, ${ }^{\mathrm{b}}$ Jan-Constantin Christopherson, ${ }^{\mathrm{a}}$ Tristan H. Borchers ${ }^{\mathrm{a}}$ \\ and Christopher J. Barrett ${ }^{\mathrm{a} *}$
}

${ }^{\mathbf{a}}$ Chemistry Department, McGill University, Montreal, Quebec, H3A 0B8, Canada, and ${ }^{\mathbf{b}}$ Department of Chemistry, Université de Montréal, Montreal, Quebec, H3C 3J7, Canada. *Correspondence e-mail: chris.barrett@mcgill.ca

The title salt, $\mathrm{C}_{8} \mathrm{H}_{22} \mathrm{~N}_{2}{ }^{2+} \cdot \mathrm{C}_{14} \mathrm{H}_{8} \mathrm{~N}_{2} \mathrm{O}_{4}{ }^{2-} \cdot \mathrm{H}_{2} \mathrm{O}$, represents a pseudo-polymer ionic material, resulting from the self-organizing behavior of $4,4^{\prime}$-azinodibenzoate dianions and doubly protonated, 1,8-diaminium-octane cations in aqueous solution. The asymmetric unit consists of two halves of octane 1,8-diaminium cations (the complete cations are both generated by crystallographic inversion symmetry), a 4,4'-azinodibenzoate anion [dihedral angle between the aromatic rings $\left.=10.22(4)^{\circ}\right]$ and a water molecule of crystallization. One of the cations is in a fully extended linear conformation while the second one has a terminal $\mathrm{C}-$ $\mathrm{C}-\mathrm{C}-\mathrm{N}$ gauche conformation. In the crystal, the cations, anions and water molecules are linked into a three-dimensional network via a complex pattern of charge-assisted $\mathrm{N}-\mathrm{H} \cdots \mathrm{O}$ and $\mathrm{O}-\mathrm{H} \cdots \mathrm{O}$ hydrogen bonds.

\section{Chemical context}

Ionic pseudo-polymers auto-assembled from oppositely charged organic molecules are of emerging interest for many potential application fields (Webber et al., 2016; Mann, 2009). It is reasonable to expect that the presence of azobenzene moieties in such materials creates the necessary precondition for achieving their reversible photosensitivity (Bushuyev et al., 2016, 2018). In this context, we report the synthesis and structure of octane-1,8-diaminium 4,4'-(diazene-1,2-diyl)dibenzoate monohydrate, (I), formed by the crystallization of bianionic 4,4'-azinodibenzoic acid and bicationic 1,8-diaminooctane in aqueous solution.<smiles>O=C([O-])c1ccc(/N=N/c2ccc(C(=O)[O-])cc2)cc1</smiles><smiles>[NH3+]CCCCCCCC[NH3+]</smiles>

\section{Structural commentary}

The asymmetric unit (Fig. 1) consists of two halves of octane 1,8-diaminium dications, one 4,4'-azinodibenzoic dianion and one water molecule. Bond lengths and angles of the dication and dianion are in the expected ranges. One of the octane 
Table 1

Selected torsion angles $\left({ }^{\circ}\right)$.

\begin{tabular}{lclr}
\hline $\mathrm{C} 2-\mathrm{C} 3-\mathrm{C} 4-\mathrm{C} 5$ & $-177.97(9)$ & $\mathrm{C} 7-\mathrm{C} 8-\mathrm{C} 9-\mathrm{C} 10$ & $-179.03(9)$ \\
$\mathrm{C} 3-\mathrm{C} 4-\mathrm{C} 5-\mathrm{C} 5^{\mathrm{i}}$ & $178.25(10)$ & $\mathrm{C} 8-\mathrm{C} 9-\mathrm{C} 10-\mathrm{C} 10^{\mathrm{ii}}$ & $178.30(11)$ \\
$\mathrm{N} 1-\mathrm{C} 2-\mathrm{C} 3-\mathrm{C} 4$ & $177.78(9)$ & $\mathrm{N} 6-\mathrm{C} 7-\mathrm{C} 8-\mathrm{C} 9$ & $-76.89(12)$ \\
\hline
\end{tabular}

Symmetry codes: (i) $-x+1,-y,-z+2$; (ii) $-x,-y+1,-z$.

Table 2

Hydrogen-bond geometry $\left(\AA,^{\circ}\right)$.

\begin{tabular}{|c|c|c|c|c|}
\hline$D-\mathrm{H} \cdots A$ & $D-\mathrm{H}$ & $\mathrm{H} \cdots A$ & $D \cdots A$ & $D-\mathrm{H} \cdots A$ \\
\hline $\mathrm{N} 1-\mathrm{H} 1 A \cdots \mathrm{O} 30^{\mathrm{iii}}$ & 0.895 (17) & 1.897 (17) & $2.7796(13)$ & $168.3(14)$ \\
\hline $\mathrm{N} 1-\mathrm{H} 1 B \cdots \mathrm{O} 13$ & $0.916(16)$ & $1.881(17)$ & $2.7942(12)$ & $175.0(14)$ \\
\hline $\mathrm{N} 1-\mathrm{H} 1 C \cdots \mathrm{O} 29^{\mathrm{iv}}$ & $0.918(16)$ & $1.970(16)$ & $2.8579(12)$ & $162.5(13)$ \\
\hline $\mathrm{N} 6-\mathrm{H} 6 A \cdots \mathrm{O} 31^{\mathrm{v}}$ & $0.921(16)$ & $1.912(16)$ & $2.8296(13)$ & $174.0(13)$ \\
\hline $\mathrm{N} 6-\mathrm{H} 6 B \cdots \mathrm{O} 11$ & $0.921(16)$ & $1.879(16)$ & $2.7735(12)$ & $163.2(13)$ \\
\hline $\mathrm{N} 6-\mathrm{H} 6 C \cdots \mathrm{O} 29^{\mathrm{vi}}$ & $0.947(16)$ & 1.849 (17) & $2.7847(12)$ & $169.0(14)$ \\
\hline $\mathrm{N} 6-\mathrm{H} 6 C \cdots \mathrm{O} 30^{\mathrm{vi}}$ & $0.947(16)$ & $2.590(16)$ & $3.0880(12)$ & $113.2(11)$ \\
\hline $\mathrm{O} 31-\mathrm{H} 31 A \cdots \mathrm{O} 13^{\mathrm{vii}}$ & $0.89(2)$ & $1.88(2)$ & $2.7418(11)$ & $165.2(17)$ \\
\hline $\mathrm{O} 31-\mathrm{H} 31 B \cdots \mathrm{O} 11$ & $0.88(2)$ & $1.97(2)$ & $2.8184(11)$ & $162.2(16)$ \\
\hline
\end{tabular}

Symmetry codes: (iii) $\quad-x+1,-y+1,-z$; $\quad$ (iv) $\quad x+1, y-1, z+1$; $-x,-y+1,-z+1$; (vi) $x, y-1, z+1$; (vii) $-x+1,-y+1,-z+1$.

1,8-diaminium dications shows a fully extended all-trans conformation with torsion angles close to $180^{\circ}$ (Table 1$)$. The second cation has its two terminal torsion angles $\mathrm{N} 6-\mathrm{C} 7-$ C8-C9 synclinal with a value of $-76.89(12)^{\circ}$. The fully extended conformation corresponds to the most stable one, compared to the arrangement with synclinal torsion angles, as shown from DFT calculations and a database survey performed on $\alpha, \omega$-alkyldiaminium cations (Brozdowska \& Chojnacki, 2017). The less energetically favorable gauche conformation is presumably stabilized by the charge-assisted hydrogen-bonded network.

The geometry of the $4,4^{\prime}$-azinodibenzoic dianion is characterized by the presence of two benzoic acid residues linked via a trans-configurated azo group is consistent with known data (Fernández et al., 2015; Sahoo et al., 2012). The angle between the phenyl rings of $10.22(4)^{\circ}$ is consistent with a small deviation from planarity of the azobenzene moiety. The carboxylate groups are inclined to the molecular mean plane at angles of $38.40(3)^{\circ}(\mathrm{O} 11 / \mathrm{C} 12 / \mathrm{O} 13)$ and $16.53(5)^{\circ}(\mathrm{O} 29 /$ C28/O30).

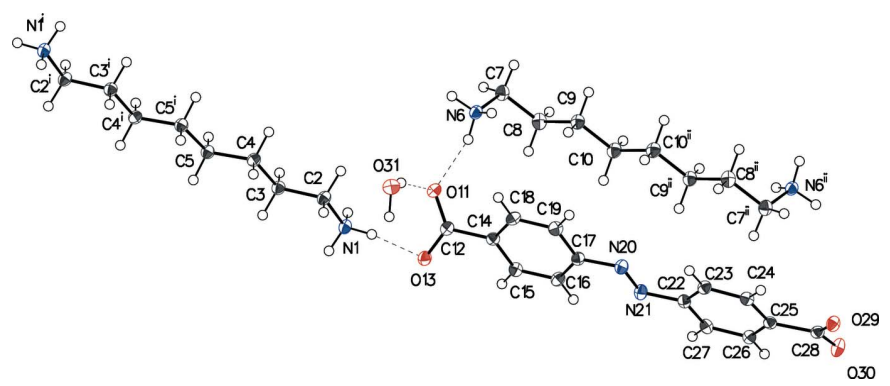

Figure 1

Molecular structure and atom-labelling scheme of (I). Displacement ellipsoids are drawn at the $50 \%$ probability level and hydrogen bonds are shown as dotted lines. [Symmetry codes: (i) $1-x,-y, 2-z$; (ii) $-x, 1-y$, $-z$.

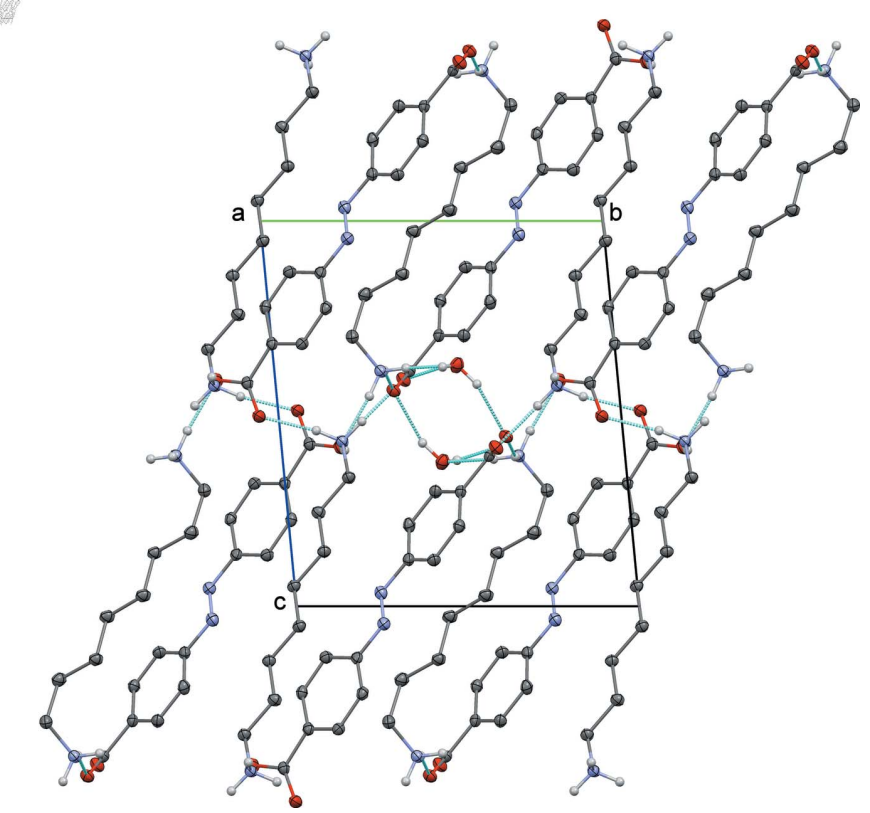

Figure 2

Projection along the $b$-axis direction showing the packing in layers consolidated by the hydrogen-bond network (dotted lines). Hydrogen atoms not involved in hydrogen bonds and hanging hydrogen bonds are omitted for clarity.

\section{Supramolecular features}

In addition to the electrostatic interactions, the anions and cations are connected by charge-assisted $\mathrm{N}-\mathrm{H}$. . O hydrogen bonds (Table 2). The complex pattern of hydrogen bonds also includes the water molecules. Therefore, the 4,4'-azinodibenzoic dianion is linked through hydrogen bonds with three cations on one side and with two cations and two water molecules on the other side. Anions and cation stack in twodimensional arrays in the $a b$ plane separated by a zone with

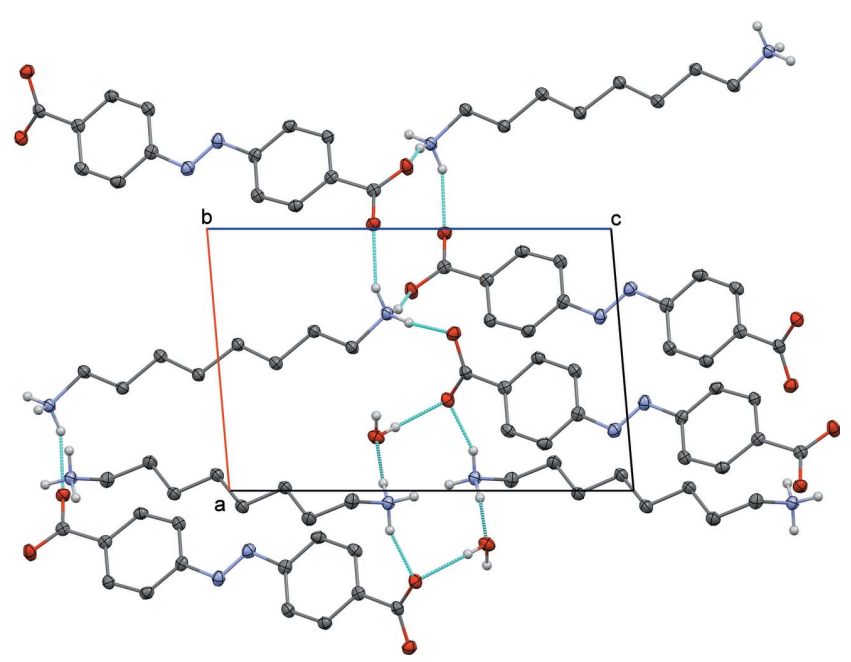

Figure 3

Partial packing view along the $b$-axis direction showing the $R_{4}^{4}(12)$ graphset motifs. Hydrogen atoms not involved in hydrogen bonds and hanging hydrogen bonds have been omitted for clarity. 
Table 3

Experimental details.

\begin{tabular}{|c|c|}
\hline \multicolumn{2}{|l|}{ Crystal data } \\
\hline Chemical formula & $\mathrm{C}_{8} \mathrm{H}_{22} \mathrm{~N}_{2}{ }^{2+} \cdot \mathrm{C}_{14} \mathrm{H}_{8} \mathrm{~N}_{2} \mathrm{O}_{4}{ }^{2-} \cdot \mathrm{H}_{2} \mathrm{O}$ \\
\hline$M_{\mathrm{r}}$ & 432.51 \\
\hline Crystal system, space group & Triclinic, $P \overline{1}$ \\
\hline Temperature $(\mathrm{K})$ & 100 \\
\hline$a, b, c(\AA)$ & $8.3604(1), 11.4100(2), 12.4661$ (2) \\
\hline$\alpha, \beta, \gamma\left(^{\circ}\right)$ & $83.001(1), 83.364(1), 73.973$ (1) \\
\hline$V\left(\AA^{3}\right)$ & $1130.24(3)$ \\
\hline$Z$ & 2 \\
\hline Radiation type & $\mathrm{Cu} K \alpha$ \\
\hline$\mu\left(\mathrm{mm}^{-1}\right)$ & 0.75 \\
\hline Crystal size (mm) & $0.4 \times 0.4 \times 0.1$ \\
\hline \multicolumn{2}{|l|}{ Data collection } \\
\hline Diffractometer & Bruker APEXII CCD \\
\hline Absorption correction & $\begin{array}{l}\text { Multi-scan (SADABS; Krause et } \\
\quad \text { al., 2015) }\end{array}$ \\
\hline$T_{\min }, T_{\max }$ & $0.525,0.753$ \\
\hline $\begin{array}{l}\text { No. of measured, independent and } \\
\text { observed }[I>2 \sigma(I)] \text { reflections }\end{array}$ & $28413,4048,3920$ \\
\hline$R_{\text {int }}$ & 0.046 \\
\hline$(\sin \theta / \lambda)_{\max }\left(\AA^{-1}\right)$ & 0.602 \\
\hline \multicolumn{2}{|l|}{ Refinement } \\
\hline$R\left[F^{2}>2 \sigma\left(F^{2}\right)\right], w R\left(F^{2}\right), S$ & $0.037,0.096,1.05$ \\
\hline No. of reflections & 4048 \\
\hline No. of parameters & 313 \\
\hline $\mathrm{H}$-atom treatment & $\begin{array}{l}\mathrm{H} \text { atoms treated by a mixture of } \\
\text { independent and constrained } \\
\text { refinement }\end{array}$ \\
\hline$\Delta \rho_{\max }, \Delta \rho_{\min }\left(\mathrm{e} \AA^{-3}\right)$ & $0.20,-0.30$ \\
\hline
\end{tabular}

Computer programs: APEX2 and SAINT (Bruker, 2013), SHELXT (Sheldrick, 2015a), SHELXL2018 (Sheldrick, 2015b), OLEX2 (Dolomanov et al., 2009), Mercury (Macrae et al., 2008) and publCIF (Westrip, 2010).

the hydrogen-bonded network involving the ionized amino and carboxylic groups and the water molecules (Fig. 2). This network contains two 12-membered rings comprising either two cations and two anions or two cations, two anions and two water molecules (Fig. 3), according to the graph set descriptor $R_{4}^{4}(12)$ (Etter et al., 1990).

\section{Database survey}

A search in the Cambridge Structural Database (Version 5.39 with one update; Groom et al., 2016) returned 48 entries for octane-1,8-diaminium compounds. These include simple halide salts (Brisson \& Brisse, 1984; van Blerk \& Kruger, 2007; van Megen \& Reiss, 2013); metal halide salts (Kessentini et al., 2011) comprising lead halide complexes (Lemmerer \& Billing, 2012; Smith et al., 2017), and more complex systems where the diaminium cations are encapsulated in a macrocycle (Kim et al., 2009; Yu et al., 2014). A similar search for 4,4'-azinodibenzoic acid and its salts returned 43 entries, including the structure of the simple acid (Yu \& Liu, 2009). The dianion has been also used as linker to prepare MOF or coordination frameworks (see, for example, Hou et al., 2013, Zhang et al., 2016, Guo et al., 2013 and Deng et al., 2015), and co-crystallized to give gelator salts (Sahoo \& Dastidar, 2012; Sahoo et al., 2012) or supramolecular assemblies (Beatty et al., 2002; Yu et al., 2011).

\section{Synthesis and crystallization}

Crystals of the title compound were obtained by the dropwise addition with intensive stirring of $5 \mathrm{ml}$ of $0.10 \mathrm{M}$ aqueous 1,8 octamethylenediamine into $25 \mathrm{ml}$ of $0.02 \mathrm{M}$ aqueous $4,4^{\prime}$-dicarboxyazobenzene disodium salt at room temperature. The final solution ( $\mathrm{pH}$ 12.5) was allowed to partly evaporate at room temperature and atmospheric pressure. The resulting orange oblong crystals in the form of thin narrow leaves up to $1 \mathrm{~cm}$ long were gently removed from the liquid phase and airdried on filter paper.

\section{Refinement}

Crystal data, data collection and structure refinement details are summarized in Table 3. Hydrogen atoms bound to nitrogen or oxygen atoms were located from difference syntheses and refined without any restraints. Hydrogen atoms linked to carbon atoms were included using an appropriate riding model (AFIX 43 and AFIX 23 for aromatic and methylene hydrogen atoms respectively) with $\mathrm{C}-\mathrm{H}=0.95-0.99 \AA$ and $U_{\text {iso }}(\mathrm{H})=1.2 U_{\text {eq }}(\mathrm{C})$.

\section{Acknowledgements}

Prof. Tomislav Friščić and Dr Hatem M. Titi are gratefully acknowledged for their help and valuable advice related to this project. The Fonds de Recherche du Québec - Nature et Technologies is gratefully acknowledged for a postdoctoral fellowship to IE.

\section{References}

Beatty, A. M., Schneider, C. M., Simpson, A. E. \& Zaher, J. L. (2002). CrystEngComm, 4, 282-287.

Blerk, C. van \& Kruger, G. J. (2007). Acta Cryst. E63, o4289.

Brisson, J. \& Brisse, F. (1984). Acta Cryst. C40, 1405-1407.

Brozdowska, A. \& Chojnacki, J. (2017). Acta Cryst. B73, 507-518.

Bruker (2013). APEX2 and SAINT. Bruker AXS Inc., Madison, Wisconsin, USA.

Bushuyev, O. S., Aizawa, M., Shishido, A. \& Barrett, C. J. (2018). Macromol. Rapid Commun. 39, 1700253.

Bushuyev, O. S., Friščić, T. \& Barrett, C. J. (2016). CrystEngComm, 18, 7204-7211.

Deng, M., Tai, S., Zhang, W., Wang, Y., Zhu, J., Zhang, J., Ling, Y. \& Zhou, Y. (2015). CrystEngComm, 17, 6023-6029.

Dolomanov, O. V., Bourhis, L. J., Gildea, R. J., Howard, J. A. K. \& Puschmann, H. (2009). J. Appl. Cryst. 42, 339-341.

Etter, M. C., MacDonald, J. C. \& Bernstein, J. (1990). Acta Cryst. B46, 256-262.

Fernández, B., Seco, J. M., Cepeda, J., Calahorro, A. J. \& RodríguezDiéguez, A. (2015). CrystEngComm, 17, 7636-7645.

Groom, C. R., Bruno, I. J., Lightfoot, M. P. \& Ward, S. C. (2016). Acta Cryst. B72, 171-179.

Guo, J. S., Xu, G., Guo, G.-C. \& Huang, J.-S. (2013). Cryst. Growth Des. 13, 2642-2649.

Hou, Y.-F., Yu, Y., Yue, K.-F., Wei, Q., Liu, Y., Zhou, C.-S. \& Wang, Y.-Y. (2013). CrystEngComm, 15, 7161-7165.

Kessentini, A., Belhouchet, M. \& Mhiri, T. (2011). X-ray Struct. Anal. Online, 27, 31-32.

Kim, Y., Kim, H., Ko, Y. H., Selvapalam, N., Rekharsky, M. V., Inoue, Y. \& Kim, K. (2009). Chem. Eur. J. 15, 6143-6151. 
Krause, L., Herbst-Irmer, R., Sheldrick, G. M. \& Stalke, D. (2015). J. Appl. Cryst. 48, 3-10.

Lemmerer, A. \& Billing, D. G. (2012). CrystEngComm, 14, 1954 1966.

Macrae, C. F., Bruno, I. J., Chisholm, J. A., Edgington, P. R., McCabe, P., Pidcock, E., Rodriguez-Monge, L., Taylor, R., van de Streek, J. \& Wood, P. A. (2008). J. Appl. Cryst. 41, 466-470.

Mann, S. (2009). Nat. Mater. 8, 781-792.

Megen, M. van \& Reiss, G. J. (2013). Inorganic 1, 3-13.

Sahoo, P., Chakraborty, I. \& Dastidar, P. (2012). Soft Matter, 8, 25952598.

Sahoo, P. \& Dastidar, P. (2012). Cryst. Growth Des. 12, 5917-5924.

Sheldrick, G. M. (2015a). Acta Cryst. A71, 3-8.
Sheldrick, G. M. (2015b). Acta Cryst. C71, 3-8.

Smith, M. D., Jaffe, A., Dohner, E. R., Lindenberg, A. M. \& Karunadasa, H. I. (2017). Chem. Sci. 8, 4497-4504.

Webber, M. J., Appel, E. A., Meijer, E. W. \& Langer, R. (2016). Nat. Mater. 15, 13-26.

Westrip, S. P. (2010). J. Appl. Cryst. 43, 920-925.

Yu, G., Hua, B. \& Han, C. (2014). Org. Lett. 16, 2486-2489.

Yu, Q.-D. \& Liu, Y.-Y. (2009). Acta Cryst. E65, o2326.

Yu, Z. B., Sun, J., Huang, Z. T. \& Zheng, Q. Y. (2011). CrystEngComm, 13, 1287-1290.

Zhang, L., Feng, X. F., Meng, P. P., Gong, L. L. \& Luo, F. (2016). CrystEngComm, 18, 1693-1698. 


\section{supporting information}

Acta Cryst. (2018). E74, 724-727 [https://doi.org/10.1107/S2056989018006187]

\section{Crystal structure of octane-1,8-diaminium 4,4'-(diazene-1,2-diyl)dibenzoate monohydrate}

Igor Elkin, Thierry Maris, Jan-Constantin Christopherson, Tristan H. Borchers and Christopher J. Barrett

Computing details

Data collection: APEX2 (Bruker, 2013); cell refinement: SAINT (Bruker, 2013); data reduction: SAINT (Bruker, 2013); program(s) used to solve structure: SHELXT (Sheldrick, 2015a); program(s) used to refine structure: SHELXL2018 (Sheldrick, 2015b); molecular graphics: OLEX2 (Dolomanov et al., 2009) and Mercury (Macrae et al., 2008); software used to prepare material for publication: OLEX2 (Dolomanov et al., 2009) and publCIF (Westrip, 2010).

Octane-1,8-diaminium 4,4'-(diazene-1,2-diyl)dibenzoate monohydrate

Crystal data

$\mathrm{C}_{8} \mathrm{H}_{22} \mathrm{~N}_{2}{ }^{2+} \cdot \mathrm{C}_{14} \mathrm{H}_{8} \mathrm{~N}_{2} \mathrm{O}_{4}{ }^{2-} \cdot \mathrm{H}_{2} \mathrm{O}$

$M_{r}=432.51$

Triclinic, $P \overline{1}$

$a=8.3604(1) \AA$

$b=11.4100(2) \AA$

$c=12.4661(2) \AA$

$\alpha=83.001(1)^{\circ}$

$\beta=83.364(1)^{\circ}$

$\gamma=73.973(1)^{\circ}$

$V=1130.24(3) \AA^{3}$

Data collection

Bruker APEXII CCD

diffractometer

Radiation source: fine-focus sealed tube

Graphite monochromator

$\varphi$ and $\omega$ scans

Absorption correction: multi-scan

(SADABS; Krause et al., 2015)

$T_{\min }=0.525, T_{\max }=0.753$

Refinement

Refinement on $F^{2}$

Least-squares matrix: full

$R\left[F^{2}>2 \sigma\left(F^{2}\right)\right]=0.037$

$w R\left(F^{2}\right)=0.096$

$S=1.05$

4048 reflections

313 parameters
$Z=2$

$F(000)=464$

$D_{\mathrm{x}}=1.271 \mathrm{Mg} \mathrm{m}^{-3}$

$\mathrm{Cu} K \alpha$ radiation, $\lambda=1.54184 \AA$

Cell parameters from 3920 reflections

$\theta=4.1-68.1^{\circ}$

$\mu=0.75 \mathrm{~mm}^{-1}$

$T=100 \mathrm{~K}$

Block, orange

$0.4 \times 0.4 \times 0.1 \mathrm{~mm}$

28413 measured reflections

4048 independent reflections

3920 reflections with $I>2 \sigma(I)$

$R_{\text {int }}=0.046$

$\theta_{\max }=68.1^{\circ}, \theta_{\min }=4.1^{\circ}$

$h=-10 \rightarrow 10$

$k=-13 \rightarrow 13$

$l=-14 \rightarrow 14$

0 restraints

Primary atom site location: structure-invariant direct methods

Hydrogen site location: mixed

$\mathrm{H}$ atoms treated by a mixture of independent and constrained refinement 
$w=1 /\left[\sigma^{2}\left(F_{\mathrm{o}}^{2}\right)+(0.0541 P)^{2}+0.3252 P\right]$

where $P=\left(F_{\mathrm{o}}^{2}+2 F_{\mathrm{c}}^{2}\right) / 3$

$(\Delta / \sigma)_{\max }=0.001$

$\Delta \rho_{\max }=0.20 \mathrm{e} \AA^{-3}$

$\Delta \rho_{\min }=-0.30$ e $\AA^{-3}$

Special details

Geometry. All esds (except the esd in the dihedral angle between two 1.s. planes) are estimated using the full covariance matrix. The cell esds are taken into account individually in the estimation of esds in distances, angles and torsion angles; correlations between esds in cell parameters are only used when they are defined by crystal symmetry. An approximate (isotropic) treatment of cell esds is used for estimating esds involving l.s. planes.

Fractional atomic coordinates and isotropic or equivalent isotropic displacement parameters $\left(\AA^{2}\right)$

\begin{tabular}{|c|c|c|c|c|}
\hline & $x$ & $y$ & $z$ & $U_{\text {iso }} * / U_{\text {eq }}$ \\
\hline C12 & $0.44977(13)$ & $0.39551(9)$ & $0.39040(8)$ & $0.0164(2)$ \\
\hline C14 & $0.39242(13)$ & $0.48992(10)$ & $0.29711(9)$ & $0.0163(2)$ \\
\hline C15 & $0.50609(13)$ & $0.50353(10)$ & $0.20773(9)$ & $0.0180(2)$ \\
\hline H15 & 0.618450 & 0.454880 & 0.207597 & $0.022 *$ \\
\hline $\mathrm{C} 16$ & $0.45684(14)$ & $0.58671(10)$ & $0.11990(9)$ & $0.0187(2)$ \\
\hline H16 & 0.534114 & 0.593956 & 0.058933 & $0.022 *$ \\
\hline $\mathrm{C} 17$ & $0.29240(14)$ & $0.66026(10)$ & $0.12125(9)$ & $0.0175(2)$ \\
\hline $\mathrm{C} 18$ & $0.17852(14)$ & $0.64902(10)$ & $0.21041(9)$ & $0.0193(2)$ \\
\hline H18 & 0.067342 & 0.699946 & 0.211663 & $0.023^{*}$ \\
\hline C19 & $0.22842(14)$ & $0.56296(10)$ & $0.29746(9)$ & $0.0185(2)$ \\
\hline H19 & 0.150301 & 0.553905 & 0.357480 & $0.022 *$ \\
\hline $\mathrm{C} 22$ & $0.27923(14)$ & $0.83834(10)$ & $-0.13411(9)$ & $0.0169(2)$ \\
\hline $\mathrm{C} 23$ & $0.12601(14)$ & $0.92810(10)$ & $-0.13255(9)$ & $0.0182(2)$ \\
\hline $\mathrm{H} 23$ & 0.049801 & 0.933790 & -0.069462 & $0.022 *$ \\
\hline $\mathrm{C} 24$ & $0.08731(13)$ & $1.00833(10)$ & $-0.22421(9)$ & $0.0174(2)$ \\
\hline $\mathrm{H} 24$ & -0.015163 & 1.070596 & -0.222999 & $0.021^{*}$ \\
\hline $\mathrm{C} 25$ & 0.19653 (13) & 0.99937 (9) & $-0.31875(9)$ & $0.0162(2)$ \\
\hline $\mathrm{C} 26$ & $0.35000(14)$ & $0.91128(10)$ & $-0.31874(9)$ & $0.0173(2)$ \\
\hline H26 & 0.426383 & 0.905667 & -0.381738 & $0.021^{*}$ \\
\hline $\mathrm{C} 27$ & $0.39101(13)$ & $0.83176(10)$ & $-0.22644(9)$ & $0.0178(2)$ \\
\hline $\mathrm{H} 27$ & 0.496160 & 0.772422 & -0.226335 & $0.021^{*}$ \\
\hline $\mathrm{C} 28$ & $0.14659(13)$ & $1.08221(10)$ & $-0.42094(9)$ & $0.0168(2)$ \\
\hline N20 & $0.23031(12)$ & $0.75035(8)$ & $0.03481(8)$ & $0.0199(2)$ \\
\hline N21 & $0.33560(12)$ & $0.75025(8)$ & $-0.04586(7)$ & $0.0194(2)$ \\
\hline O11 & $0.34625(9)$ & $0.34229(7)$ & $0.44062(6)$ & $0.0200(2)$ \\
\hline $\mathrm{O} 13$ & $0.59935(9)$ & $0.37427(7)$ & $0.41176(6)$ & $0.0209(2)$ \\
\hline O29 & $0.01715(9)$ & 1.17097 (7) & $-0.41274(6)$ & $0.0204(2)$ \\
\hline $\mathrm{O} 30$ & $0.23647(10)$ & $1.05688(7)$ & $-0.50725(6)$ & $0.0234(2)$ \\
\hline $\mathrm{C} 2$ & $0.54646(13)$ & $0.18776(10)$ & $0.66601(9)$ & $0.0181(2)$ \\
\hline $\mathrm{H} 2 \mathrm{~A}$ & 0.442430 & 0.177349 & 0.642576 & $0.022 *$ \\
\hline $\mathrm{H} 2 \mathrm{~B}$ & 0.521051 & 0.269756 & 0.692620 & $0.022 *$ \\
\hline C3 & $0.60840(13)$ & $0.08987(10)$ & $0.75674(9)$ & $0.0183(2)$ \\
\hline $\mathrm{H} 3 \mathrm{~A}$ & 0.715478 & 0.098563 & 0.776743 & $0.022^{*}$ \\
\hline H3B & 0.630505 & 0.008358 & 0.729672 & $0.022 *$ \\
\hline
\end{tabular}

Extinction correction: SHELXL2018

(Sheldrick, 2015b),

$\mathrm{Fc}^{*}=\mathrm{kFc}\left[1+0.001 \mathrm{xFc}^{2} \lambda^{3} / \sin (2 \theta)\right]^{-1 / 4}$

Extinction coefficient: 0.0427 (17) 


$\begin{array}{lllll}\text { C4 } & 0.48635(13) & 0.09539(10) & 0.85797(8) & 0.0176(2) \\ \text { H4A } & 0.380562 & 0.083043 & 0.839346 & 0.021^{*} \\ \text { H4B } & 0.461117 & 0.177451 & 0.884428 & 0.021^{*} \\ \text { C5 } & 0.55783(13) & -0.00224(10) & 0.94788(8) & 0.0178(2) \\ \text { H5A } & 0.580205 & -0.084114 & 0.921493 & 0.021^{*} \\ \text { H5B } & 0.665684 & 0.008692 & 0.964131 & 0.021^{*} \\ \text { N1 } & 0.67578(12) & 0.17874(9) & 0.57222(8) & 0.0177(2) \\ \text { H1A } & 0.6930(19) & 0.1068(16) & 0.5450(12) & 0.033(4)^{*} \\ \text { H1B } & 0.6457(19) & 0.2412(15) & 0.5188(12) & 0.031(4)^{*} \\ \text { H1C } & 0.777(2) & 0.1830(13) & 0.5905(12) & 0.028(4)^{*} \\ \text { C7 } & 0.04089(14) & 0.23686(10) & 0.30051(9) & 0.0206(3) \\ \text { H7A } & -0.076237 & 0.235667 & 0.296577 & 0.025^{*} \\ \text { H7B } & 0.107206 & 0.151394 & 0.317135 & 0.025^{*} \\ \text { C8 } & 0.10729(14) & 0.28667(10) & 0.19086(9) & 0.0222(3) \\ \text { H8A } & 0.134670 & 0.221260 & 0.141092 & 0.027^{*} \\ \text { H8B } & 0.212435 & 0.306963 & 0.199818 & 0.027^{*} \\ \text { C9 } & -0.01161(13) & 0.39982(10) & 0.13799(9) & 0.0194(2) \\ \text { H9A } & -0.117543 & 0.380517 & 0.129346 & 0.023^{*} \\ \text { H9B } & -0.037363 & 0.466406 & 0.186456 & 0.023^{*} \\ \text { C10 } & 0.06004(14) & 0.44447(10) & 0.02760(9) & 0.0210(3) \\ \text { H10A } & 0.089089 & 0.376931 & -0.020020 & 0.025^{*} \\ \text { H10B } & 0.164268 & 0.465977 & 0.036703 & 0.025^{*} \\ \text { N6 } & 0.04744(12) & 0.31029(9) & 0.39017(7) & 0.0178(2) \\ \text { H6A } & -0.0289(18) & 0.3860(14) & 0.3860(11) & 0.026(3)^{*} \\ \text { H6B } & 0.153(2) & 0.3200(13) & 0.3926(12) & 0.029(4)^{*} \\ \text { H6C } & 0.0223(19) & 0.2670(14) & 0.4569(13) & 0.033(4)^{*} \\ \text { O31 } & 0.20434(10) & 0.46567(8) & 0.62560(7) & 0.0235(2) \\ \text { H31A } & 0.281(2) & 0.5069(17) & 0.6193(14) & 0.049(5)^{*} \\ \text { H31B } & 0.241(2) & 0.4140(17) & 0.5755(15) & 0.048(5)^{*} \\ & & & & \end{array}$

Atomic displacement parameters $\left(\AA^{2}\right)$

\begin{tabular}{lllllll}
\hline & $U^{11}$ & $U^{22}$ & $U^{33}$ & $U^{12}$ & $U^{13}$ & $U^{23}$ \\
\hline C12 & $0.0197(6)$ & $0.0127(5)$ & $0.0160(5)$ & $-0.0030(4)$ & $0.0010(4)$ & $-0.0038(4)$ \\
C14 & $0.0187(5)$ & $0.0134(5)$ & $0.0176(5)$ & $-0.0050(4)$ & $-0.0011(4)$ & $-0.0029(4)$ \\
C15 & $0.0165(5)$ & $0.0163(5)$ & $0.0192(5)$ & $-0.0014(4)$ & $-0.0007(4)$ & $-0.0022(4)$ \\
C16 & $0.0201(6)$ & $0.0182(5)$ & $0.0170(5)$ & $-0.0046(4)$ & $0.0020(4)$ & $-0.0024(4)$ \\
C17 & $0.0209(6)$ & $0.0135(5)$ & $0.0184(5)$ & $-0.0048(4)$ & $-0.0030(4)$ & $-0.0012(4)$ \\
C18 & $0.0160(5)$ & $0.0161(5)$ & $0.0234(6)$ & $-0.0009(4)$ & $-0.0009(4)$ & $-0.0015(4)$ \\
C19 & $0.0188(5)$ & $0.0161(5)$ & $0.0197(5)$ & $-0.0048(4)$ & $0.0029(4)$ & $-0.0017(4)$ \\
C22 & $0.0203(5)$ & $0.0137(5)$ & $0.0174(5)$ & $-0.0041(4)$ & $-0.0041(4)$ & $-0.0023(4)$ \\
C23 & $0.0193(5)$ & $0.0183(5)$ & $0.0168(5)$ & $-0.0053(4)$ & $0.0003(4)$ & $-0.0027(4)$ \\
C24 & $0.0156(5)$ & $0.0147(5)$ & $0.0210(5)$ & $-0.0017(4)$ & $-0.0020(4)$ & $-0.0026(4)$ \\
C25 & $0.0177(5)$ & $0.0136(5)$ & $0.0183(5)$ & $-0.0051(4)$ & $-0.0028(4)$ & $-0.0021(4)$ \\
C26 & $0.0186(5)$ & $0.0171(5)$ & $0.0164(5)$ & $-0.0044(4)$ & $-0.0005(4)$ & $-0.0034(4)$ \\
C27 & $0.0170(5)$ & $0.0155(5)$ & $0.0198(5)$ & $-0.0006(4)$ & $-0.0036(4)$ & $-0.0036(4)$ \\
C28 & $0.0180(5)$ & $0.0147(5)$ & $0.0186(5)$ & $-0.0057(4)$ & $-0.0017(4)$ & $-0.0014(4)$ \\
N20 & $0.0213(5)$ & $0.0176(5)$ & $0.0197(5)$ & $-0.0042(4)$ & $-0.0027(4)$ & $0.0006(4)$
\end{tabular}


Geometric parameters $\left(\AA,{ }^{\circ}\right)$

\begin{tabular}{llll}
\hline $\mathrm{C} 12-\mathrm{C} 14$ & $1.5103(14)$ & $\mathrm{C} 2-\mathrm{N} 1$ & $1.4907(13)$ \\
$\mathrm{C} 12-\mathrm{O} 11$ & $1.2578(13)$ & $\mathrm{C} 3-\mathrm{H} 3 \mathrm{~A}$ & 0.9900 \\
$\mathrm{C} 12-\mathrm{O} 13$ & $1.2599(14)$ & $\mathrm{C} 3-\mathrm{H} 3 \mathrm{~B}$ & 0.9900 \\
$\mathrm{C} 14-\mathrm{C} 15$ & $1.4011(15)$ & $\mathrm{C} 3-\mathrm{C} 4$ & $1.5244(14)$ \\
$\mathrm{C} 14-\mathrm{C} 19$ & $1.3939(15)$ & $\mathrm{C} 4-\mathrm{H} 4 \mathrm{~A}$ & 0.9900 \\
$\mathrm{C} 15-\mathrm{H} 15$ & 0.9500 & $\mathrm{C} 4-\mathrm{H} 4 \mathrm{~B}$ & 0.9900 \\
$\mathrm{C} 15-\mathrm{C} 16$ & $1.3800(15)$ & $\mathrm{C} 4-\mathrm{C} 5$ & $1.5289(15)$ \\
$\mathrm{C} 16-\mathrm{H} 16$ & 0.9500 & $\mathrm{C} 5-\mathrm{C} 5$ & $1.525(2)$ \\
$\mathrm{C} 16-\mathrm{C} 17$ & $1.3984(16)$ & $\mathrm{C} 5-\mathrm{H} 5 \mathrm{~A}$ & 0.9900 \\
$\mathrm{C} 17-\mathrm{C} 18$ & $1.3951(15)$ & $\mathrm{C} 5-\mathrm{H} 5 \mathrm{~B}$ & 0.9900 \\
$\mathrm{C} 17-\mathrm{N} 20$ & $1.4325(14)$ & $\mathrm{N} 1-\mathrm{H} 1 \mathrm{~A}$ & $0.895(17)$ \\
$\mathrm{C} 18-\mathrm{H} 18$ & 0.9500 & $\mathrm{~N} 1-\mathrm{H} 1 \mathrm{~B}$ & $0.916(16)$ \\
$\mathrm{C} 18-\mathrm{C} 19$ & $1.3909(16)$ & $\mathrm{N} 1-\mathrm{H} 1 \mathrm{C}$ & $0.918(16)$ \\
$\mathrm{C} 19-\mathrm{H} 19$ & 0.9500 & $\mathrm{C} 7-\mathrm{H} 7 \mathrm{~A}$ & 0.9900 \\
$\mathrm{C} 22-\mathrm{C} 23$ & $1.4018(16)$ & $\mathrm{C} 7-\mathrm{H} 7 \mathrm{~B}$ & 0.9900 \\
$\mathrm{C} 22-\mathrm{C} 27$ & $1.3920(15)$ & $\mathrm{C} 7-\mathrm{C} 8$ & $1.5196(16)$ \\
$\mathrm{C} 22-\mathrm{N} 21$ & $1.4253(14)$ & $\mathrm{C} 7-\mathrm{N} 6$ & $1.4905(14)$ \\
$\mathrm{C} 23-\mathrm{H} 23$ & 0.9500 & $\mathrm{C} 8-\mathrm{H} 8 \mathrm{~A}$ & 0.9900 \\
$\mathrm{C} 23-\mathrm{C} 24$ & $1.3839(16)$ & $\mathrm{C} 8-\mathrm{H} 8 \mathrm{~B}$ & 0.9900 \\
$\mathrm{C} 24-\mathrm{H} 24$ & 0.9500 & $\mathrm{C} 8-\mathrm{C} 9$ & $1.5247(15)$ \\
$\mathrm{C} 24-\mathrm{C} 25$ & $1.4008(15)$ & $\mathrm{C} 9-\mathrm{H} 9 \mathrm{~A}$ & 0.9900 \\
$\mathrm{C} 25-\mathrm{C} 26$ & $1.3944(16)$ & $\mathrm{C} 9-\mathrm{H} 9 \mathrm{~B}$ & 0.9900 \\
$\mathrm{C} 25-\mathrm{C} 28$ & $1.5172(15)$ & $\mathrm{C} 9-\mathrm{C} 10$ & $1.5228(15)$ \\
$\mathrm{C} 26-\mathrm{H} 26$ & 0.9500 & $\mathrm{C} 10-\mathrm{C} 10^{\mathrm{ii}}$ & $1.527(2)$ \\
$\mathrm{C} 26-\mathrm{C} 27$ & $1.3891(15)$ & $\mathrm{C} 10-\mathrm{H} 10 \mathrm{~A}$ & 0.9900 \\
$\mathrm{C} 27-\mathrm{H} 27$ & 0.9500 & $\mathrm{C} 10-\mathrm{H} 10 \mathrm{~B}$ & 0.9900 \\
$\mathrm{C} 28-\mathrm{O} 29$ & $1.2656(13)$ & $\mathrm{N} 6-\mathrm{H} 6 \mathrm{~A}$ & $0.921(16)$ \\
$\mathrm{C} 28-\mathrm{O} 30$ & $1.2549(13)$ & $\mathrm{N} 6-\mathrm{H} 6 \mathrm{~B}$ & $0.947(16)$ \\
$\mathrm{N} 20-\mathrm{N} 21$ & $1.2575(14)$ & $\mathrm{N} 6-\mathrm{H} 6 \mathrm{C}$ &
\end{tabular}




\begin{tabular}{|c|c|c|c|}
\hline $\mathrm{C} 2-\mathrm{H} 2 \mathrm{~A}$ & 0.9900 & $\mathrm{O} 31-\mathrm{H} 31 \mathrm{~A}$ & $0.89(2)$ \\
\hline $\mathrm{C} 2-\mathrm{H} 2 \mathrm{~B}$ & 0.9900 & $\mathrm{O} 31-\mathrm{H} 31 \mathrm{~B}$ & $0.88(2)$ \\
\hline $\mathrm{C} 2-\mathrm{C} 3$ & $1.5176(15)$ & & \\
\hline $\mathrm{O} 11-\mathrm{C} 12-\mathrm{C} 14$ & $118.09(9)$ & $\mathrm{H} 3 \mathrm{~A}-\mathrm{C} 3-\mathrm{H} 3 \mathrm{~B}$ & 107.7 \\
\hline $\mathrm{O} 11-\mathrm{C} 12-\mathrm{O} 13$ & $124.36(10)$ & $\mathrm{C} 4-\mathrm{C} 3-\mathrm{H} 3 \mathrm{~A}$ & 108.8 \\
\hline $\mathrm{O} 13-\mathrm{C} 12-\mathrm{C} 14$ & $117.54(9)$ & $\mathrm{C} 4-\mathrm{C} 3-\mathrm{H} 3 \mathrm{~B}$ & 108.8 \\
\hline $\mathrm{C} 15-\mathrm{C} 14-\mathrm{C} 12$ & $119.29(9)$ & $\mathrm{C} 3-\mathrm{C} 4-\mathrm{H} 4 \mathrm{~A}$ & 109.3 \\
\hline $\mathrm{C} 19-\mathrm{C} 14-\mathrm{C} 12$ & $121.45(9)$ & $\mathrm{C} 3-\mathrm{C} 4-\mathrm{H} 4 \mathrm{~B}$ & 109.3 \\
\hline $\mathrm{C} 19-\mathrm{C} 14-\mathrm{C} 15$ & $119.26(10)$ & $\mathrm{C} 3-\mathrm{C} 4-\mathrm{C} 5$ & $111.41(9)$ \\
\hline $\mathrm{C} 14-\mathrm{C} 15-\mathrm{H} 15$ & 119.6 & $\mathrm{H} 4 \mathrm{~A}-\mathrm{C} 4-\mathrm{H} 4 \mathrm{~B}$ & 108.0 \\
\hline $\mathrm{C} 16-\mathrm{C} 15-\mathrm{C} 14$ & $120.82(10)$ & $\mathrm{C} 5-\mathrm{C} 4-\mathrm{H} 4 \mathrm{~A}$ & 109.3 \\
\hline $\mathrm{C} 16-\mathrm{C} 15-\mathrm{H} 15$ & 119.6 & $\mathrm{C} 5-\mathrm{C} 4-\mathrm{H} 4 \mathrm{~B}$ & 109.3 \\
\hline $\mathrm{C} 15-\mathrm{C} 16-\mathrm{H} 16$ & 120.2 & $\mathrm{C} 4-\mathrm{C} 5-\mathrm{H} 5 \mathrm{~A}$ & 108.9 \\
\hline $\mathrm{C} 15-\mathrm{C} 16-\mathrm{C} 17$ & $119.54(10)$ & $\mathrm{C} 4-\mathrm{C} 5-\mathrm{H} 5 \mathrm{~B}$ & 108.9 \\
\hline $\mathrm{C} 17-\mathrm{C} 16-\mathrm{H} 16$ & 120.2 & $\mathrm{C} 5^{\mathrm{i}}-\mathrm{C} 5-\mathrm{C} 4$ & $113.31(11)$ \\
\hline $\mathrm{C} 16-\mathrm{C} 17-\mathrm{N} 20$ & $123.64(10)$ & $\mathrm{C} 55^{\mathrm{i}}-\mathrm{C} 5-\mathrm{H} 5 \mathrm{~A}$ & 108.9 \\
\hline $\mathrm{C} 18-\mathrm{C} 17-\mathrm{C} 16$ & $120.26(10)$ & $\mathrm{C} 55^{\mathrm{i}}-\mathrm{C} 5-\mathrm{H} 5 \mathrm{~B}$ & 108.9 \\
\hline $\mathrm{C} 18-\mathrm{C} 17-\mathrm{N} 20$ & $116.10(9)$ & $\mathrm{H} 5 \mathrm{~A}-\mathrm{C} 5-\mathrm{H} 5 \mathrm{~B}$ & 107.7 \\
\hline $\mathrm{C} 17-\mathrm{C} 18-\mathrm{H} 18$ & 120.1 & $\mathrm{C} 2-\mathrm{N} 1-\mathrm{H} 1 \mathrm{~A}$ & $109.6(10)$ \\
\hline $\mathrm{C} 19-\mathrm{C} 18-\mathrm{C} 17$ & $119.74(10)$ & $\mathrm{C} 2-\mathrm{N} 1-\mathrm{H} 1 \mathrm{~B}$ & $112.0(9)$ \\
\hline $\mathrm{C} 19-\mathrm{C} 18-\mathrm{H} 18$ & 120.1 & $\mathrm{C} 2-\mathrm{N} 1-\mathrm{H} 1 \mathrm{C}$ & $113.2(9)$ \\
\hline $\mathrm{C} 14-\mathrm{C} 19-\mathrm{H} 19$ & 119.8 & $\mathrm{H} 1 \mathrm{~A}-\mathrm{N} 1-\mathrm{H} 1 \mathrm{~B}$ & $109.5(13)$ \\
\hline $\mathrm{C} 18-\mathrm{C} 19-\mathrm{C} 14$ & $120.36(10)$ & $\mathrm{H} 1 \mathrm{~A}-\mathrm{N} 1-\mathrm{H} 1 \mathrm{C}$ & $107.0(13)$ \\
\hline $\mathrm{C} 18-\mathrm{C} 19-\mathrm{H} 19$ & 119.8 & $\mathrm{H} 1 \mathrm{~B}-\mathrm{N} 1-\mathrm{H} 1 \mathrm{C}$ & $105.4(13)$ \\
\hline $\mathrm{C} 23-\mathrm{C} 22-\mathrm{N} 21$ & $125.14(10)$ & $\mathrm{H} 7 \mathrm{~A}-\mathrm{C} 7-\mathrm{H} 7 \mathrm{~B}$ & 107.8 \\
\hline $\mathrm{C} 27-\mathrm{C} 22-\mathrm{C} 23$ & $120.04(10)$ & $\mathrm{C} 8-\mathrm{C} 7-\mathrm{H} 7 \mathrm{~A}$ & 109.0 \\
\hline $\mathrm{C} 27-\mathrm{C} 22-\mathrm{N} 21$ & $114.81(9)$ & $\mathrm{C} 8-\mathrm{C} 7-\mathrm{H} 7 \mathrm{~B}$ & 109.0 \\
\hline $\mathrm{C} 22-\mathrm{C} 23-\mathrm{H} 23$ & 120.5 & $\mathrm{~N} 6-\mathrm{C} 7-\mathrm{H} 7 \mathrm{~A}$ & 109.0 \\
\hline $\mathrm{C} 24-\mathrm{C} 23-\mathrm{C} 22$ & $119.07(10)$ & N6-C7-H7B & 109.0 \\
\hline $\mathrm{C} 24-\mathrm{C} 23-\mathrm{H} 23$ & 120.5 & $\mathrm{~N} 6-\mathrm{C} 7-\mathrm{C} 8$ & $112.95(9)$ \\
\hline $\mathrm{C} 23-\mathrm{C} 24-\mathrm{H} 24$ & 119.4 & $\mathrm{C} 7-\mathrm{C} 8-\mathrm{H} 8 \mathrm{~A}$ & 108.5 \\
\hline $\mathrm{C} 23-\mathrm{C} 24-\mathrm{C} 25$ & $121.19(10)$ & $\mathrm{C} 7-\mathrm{C} 8-\mathrm{H} 8 \mathrm{~B}$ & 108.5 \\
\hline $\mathrm{C} 25-\mathrm{C} 24-\mathrm{H} 24$ & 119.4 & $\mathrm{C} 7-\mathrm{C} 8-\mathrm{C} 9$ & $114.99(9)$ \\
\hline $\mathrm{C} 24-\mathrm{C} 25-\mathrm{C} 28$ & $120.42(9)$ & $\mathrm{H} 8 \mathrm{~A}-\mathrm{C} 8-\mathrm{H} 8 \mathrm{~B}$ & 107.5 \\
\hline $\mathrm{C} 26-\mathrm{C} 25-\mathrm{C} 24$ & $119.24(10)$ & $\mathrm{C} 9-\mathrm{C} 8-\mathrm{H} 8 \mathrm{~A}$ & 108.5 \\
\hline $\mathrm{C} 26-\mathrm{C} 25-\mathrm{C} 28$ & $120.32(10)$ & $\mathrm{C} 9-\mathrm{C} 8-\mathrm{H} 8 \mathrm{~B}$ & 108.5 \\
\hline $\mathrm{C} 25-\mathrm{C} 26-\mathrm{H} 26$ & 120.1 & $\mathrm{C} 8-\mathrm{C} 9-\mathrm{H} 9 \mathrm{~A}$ & 109.0 \\
\hline $\mathrm{C} 27-\mathrm{C} 26-\mathrm{C} 25$ & $119.89(10)$ & $\mathrm{C} 8-\mathrm{C} 9-\mathrm{H} 9 \mathrm{~B}$ & 109.0 \\
\hline $\mathrm{C} 27-\mathrm{C} 26-\mathrm{H} 26$ & 120.1 & $\mathrm{H} 9 \mathrm{~A}-\mathrm{C} 9-\mathrm{H} 9 \mathrm{~B}$ & 107.8 \\
\hline $\mathrm{C} 22-\mathrm{C} 27-\mathrm{H} 27$ & 119.7 & $\mathrm{C} 10-\mathrm{C} 9-\mathrm{C} 8$ & $112.75(9)$ \\
\hline $\mathrm{C} 26-\mathrm{C} 27-\mathrm{C} 22$ & $120.51(10)$ & $\mathrm{C} 10-\mathrm{C} 9-\mathrm{H} 9 \mathrm{~A}$ & 109.0 \\
\hline $\mathrm{C} 26-\mathrm{C} 27-\mathrm{H} 27$ & 119.7 & $\mathrm{C} 10-\mathrm{C} 9-\mathrm{H} 9 \mathrm{~B}$ & 109.0 \\
\hline $\mathrm{O} 29-\mathrm{C} 28-\mathrm{C} 25$ & $117.57(9)$ & $\mathrm{C} 9-\mathrm{C} 10-\mathrm{C} 10^{\mathrm{ii}}$ & $113.21(11)$ \\
\hline $\mathrm{O} 30-\mathrm{C} 28-\mathrm{C} 25$ & $117.37(9)$ & $\mathrm{C} 9-\mathrm{C} 10-\mathrm{H} 10 \mathrm{~A}$ & 108.9 \\
\hline $\mathrm{O} 30-\mathrm{C} 28-\mathrm{O} 29$ & $125.06(10)$ & $\mathrm{C} 9-\mathrm{C} 10-\mathrm{H} 10 \mathrm{~B}$ & 108.9 \\
\hline $\mathrm{N} 21-\mathrm{N} 20-\mathrm{C} 17$ & $112.88(9)$ & $\mathrm{C} 10 \mathrm{ii}-\mathrm{C} 10-\mathrm{H} 10 \mathrm{~A}$ & 108.9 \\
\hline $\mathrm{N} 20-\mathrm{N} 21-\mathrm{C} 22$ & $114.90(9)$ & $\mathrm{C} 10^{\mathrm{ii}}-\mathrm{C} 10-\mathrm{H} 10 \mathrm{~B}$ & 108.9 \\
\hline
\end{tabular}




\begin{tabular}{|c|c|c|c|}
\hline $\mathrm{H} 2 \mathrm{~A}-\mathrm{C} 2-\mathrm{H} 2 \mathrm{~B}$ & 108.1 & $\mathrm{H} 10 \mathrm{~A}-\mathrm{C} 10-\mathrm{H} 10 \mathrm{~B}$ & 107.7 \\
\hline $\mathrm{C} 3-\mathrm{C} 2-\mathrm{H} 2 \mathrm{~A}$ & 109.6 & $\mathrm{C} 7-\mathrm{N} 6-\mathrm{H} 6 \mathrm{~A}$ & $112.5(9)$ \\
\hline $\mathrm{C} 3-\mathrm{C} 2-\mathrm{H} 2 \mathrm{~B}$ & 109.6 & $\mathrm{C} 7-\mathrm{N} 6-\mathrm{H} 6 \mathrm{~B}$ & $111.9(9)$ \\
\hline $\mathrm{N} 1-\mathrm{C} 2-\mathrm{H} 2 \mathrm{~A}$ & 109.6 & $\mathrm{C} 7-\mathrm{N} 6-\mathrm{H} 6 \mathrm{C}$ & $108.3(9)$ \\
\hline $\mathrm{N} 1-\mathrm{C} 2-\mathrm{H} 2 \mathrm{~B}$ & 109.6 & $\mathrm{H} 6 \mathrm{~A}-\mathrm{N} 6-\mathrm{H} 6 \mathrm{~B}$ & $109.4(13)$ \\
\hline $\mathrm{N} 1-\mathrm{C} 2-\mathrm{C} 3$ & $110.15(9)$ & $\mathrm{H} 6 \mathrm{~A}-\mathrm{N} 6-\mathrm{H} 6 \mathrm{C}$ & $107.9(12)$ \\
\hline $\mathrm{C} 2-\mathrm{C} 3-\mathrm{H} 3 \mathrm{~A}$ & 108.8 & $\mathrm{H} 6 \mathrm{~B}-\mathrm{N} 6-\mathrm{H} 6 \mathrm{C}$ & $106.5(13)$ \\
\hline $\mathrm{C} 2-\mathrm{C} 3-\mathrm{H} 3 \mathrm{~B}$ & 108.8 & $\mathrm{H} 31 \mathrm{~A}-\mathrm{O} 31-\mathrm{H} 31 \mathrm{~B}$ & $102.9(16)$ \\
\hline $\mathrm{C} 2-\mathrm{C} 3-\mathrm{C} 4$ & $113.74(9)$ & & \\
\hline $\mathrm{C} 12-\mathrm{C} 14-\mathrm{C} 15-\mathrm{C} 16$ & $178.35(9)$ & $\mathrm{C} 25-\mathrm{C} 26-\mathrm{C} 27-\mathrm{C} 22$ & $0.71(16)$ \\
\hline $\mathrm{C} 12-\mathrm{C} 14-\mathrm{C} 19-\mathrm{C} 18$ & $-179.80(9)$ & $\mathrm{C} 26-\mathrm{C} 25-\mathrm{C} 28-\mathrm{O} 29$ & $-170.63(9)$ \\
\hline $\mathrm{C} 14-\mathrm{C} 15-\mathrm{C} 16-\mathrm{C} 17$ & $1.46(16)$ & $\mathrm{C} 26-\mathrm{C} 25-\mathrm{C} 28-\mathrm{O} 30$ & $9.78(15)$ \\
\hline $\mathrm{C} 15-\mathrm{C} 14-\mathrm{C} 19-\mathrm{C} 18$ & $-0.49(16)$ & $\mathrm{C} 27-\mathrm{C} 22-\mathrm{C} 23-\mathrm{C} 24$ & $0.85(16)$ \\
\hline $\mathrm{C} 15-\mathrm{C} 16-\mathrm{C} 17-\mathrm{C} 18$ & $-0.48(16)$ & $\mathrm{C} 27-\mathrm{C} 22-\mathrm{N} 21-\mathrm{N} 20$ & $-175.22(9)$ \\
\hline $\mathrm{C} 15-\mathrm{C} 16-\mathrm{C} 17-\mathrm{N} 20$ & $179.03(9)$ & $\mathrm{C} 28-\mathrm{C} 25-\mathrm{C} 26-\mathrm{C} 27$ & $-176.74(9)$ \\
\hline $\mathrm{C} 16-\mathrm{C} 17-\mathrm{C} 18-\mathrm{C} 19$ & $-0.97(16)$ & $\mathrm{N} 20-\mathrm{C} 17-\mathrm{C} 18-\mathrm{C} 19$ & $179.48(9)$ \\
\hline $\mathrm{C} 16-\mathrm{C} 17-\mathrm{N} 20-\mathrm{N} 21$ & $4.90(15)$ & $\mathrm{N} 21-\mathrm{C} 22-\mathrm{C} 23-\mathrm{C} 24$ & $179.78(9)$ \\
\hline $\mathrm{C} 17-\mathrm{C} 18-\mathrm{C} 19-\mathrm{C} 14$ & $1.45(16)$ & $\mathrm{N} 21-\mathrm{C} 22-\mathrm{C} 27-\mathrm{C} 26$ & $178.99(9)$ \\
\hline $\mathrm{C} 17-\mathrm{N} 20-\mathrm{N} 21-\mathrm{C} 22$ & $179.88(8)$ & $\mathrm{O} 11-\mathrm{C} 12-\mathrm{C} 14-\mathrm{C} 15$ & $-146.46(10)$ \\
\hline $\mathrm{C} 18-\mathrm{C} 17-\mathrm{N} 20-\mathrm{N} 21$ & $-175.57(9)$ & $\mathrm{O} 11-\mathrm{C} 12-\mathrm{C} 14-\mathrm{C} 19$ & $32.86(14)$ \\
\hline $\mathrm{C} 19-\mathrm{C} 14-\mathrm{C} 15-\mathrm{C} 16$ & $-0.99(16)$ & $\mathrm{O} 13-\mathrm{C} 12-\mathrm{C} 14-\mathrm{C} 15$ & $32.65(14)$ \\
\hline $\mathrm{C} 22-\mathrm{C} 23-\mathrm{C} 24-\mathrm{C} 25$ & $1.52(16)$ & $\mathrm{O} 13-\mathrm{C} 12-\mathrm{C} 14-\mathrm{C} 19$ & $-148.03(10)$ \\
\hline $\mathrm{C} 23-\mathrm{C} 22-\mathrm{C} 27-\mathrm{C} 26$ & $-1.97(16)$ & $\mathrm{C} 2-\mathrm{C} 3-\mathrm{C} 4-\mathrm{C} 5$ & $-177.97(9)$ \\
\hline $\mathrm{C} 23-\mathrm{C} 22-\mathrm{N} 21-\mathrm{N} 20$ & $5.80(15)$ & $\mathrm{C} 3-\mathrm{C} 4-\mathrm{C} 5-\mathrm{C}^{\mathrm{i}}$ & $178.25(10)$ \\
\hline $\mathrm{C} 23-\mathrm{C} 24-\mathrm{C} 25-\mathrm{C} 26$ & $-2.76(16)$ & $\mathrm{N} 1-\mathrm{C} 2-\mathrm{C} 3-\mathrm{C} 4$ & $177.78(9)$ \\
\hline $\mathrm{C} 23-\mathrm{C} 24-\mathrm{C} 25-\mathrm{C} 28$ & $175.60(9)$ & $\mathrm{C} 7-\mathrm{C} 8-\mathrm{C} 9-\mathrm{C} 10$ & $-179.03(9)$ \\
\hline $\mathrm{C} 24-\mathrm{C} 25-\mathrm{C} 26-\mathrm{C} 27$ & $1.62(15)$ & $\mathrm{C} 8-\mathrm{C} 9-\mathrm{C} 10-\mathrm{C} 10^{\mathrm{ii}}$ & $178.30(11)$ \\
\hline $\mathrm{C} 24-\mathrm{C} 25-\mathrm{C} 28-\mathrm{O} 29$ & $11.03(14)$ & $\mathrm{N} 6-\mathrm{C} 7-\mathrm{C} 8-\mathrm{C} 9$ & $-76.89(12)$ \\
\hline $\mathrm{C} 24-\mathrm{C} 25-\mathrm{C} 28-\mathrm{O} 30$ & $-168.56(10)$ & & \\
\hline
\end{tabular}

Symmetry codes: (i) $-x+1,-y,-z+2$; (ii) $-x,-y+1,-z$.

Hydrogen-bond geometry $\left(A,{ }^{\circ}\right)$

\begin{tabular}{lllll}
\hline$D-\mathrm{H} \cdots A$ & $D-\mathrm{H}$ & $\mathrm{H} \cdots A$ & $D \cdots A$ & $D-\mathrm{H} \cdots A$ \\
\hline $\mathrm{N} 1-\mathrm{H} 1 A \cdots \mathrm{O} 30^{\mathrm{iii}}$ & $0.895(17)$ & $1.897(17)$ & $2.7796(13)$ & $168.3(14)$ \\
$\mathrm{N} 1-\mathrm{H} 1 B \cdots \mathrm{O} 13$ & $0.916(16)$ & $1.881(17)$ & $2.7942(12)$ & $175.0(14)$ \\
$\mathrm{N} 1-\mathrm{H} 1 C \cdots \mathrm{O} 29^{\text {iv }}$ & $0.918(16)$ & $1.970(16)$ & $2.8579(12)$ & $162.5(13)$ \\
$\mathrm{N} 6-\mathrm{H} 6 A \cdots \mathrm{O} 31^{\mathrm{v}}$ & $0.921(16)$ & $1.912(16)$ & $2.8296(13)$ & $174.0(13)$ \\
$\mathrm{N} 6-\mathrm{H} 6 B \cdots \mathrm{O} 11$ & $0.921(16)$ & $1.879(16)$ & $2.7735(12)$ & $163.2(13)$ \\
$\mathrm{N} 6-\mathrm{H} 6 C \cdots \mathrm{O} 299^{\text {vi }}$ & $0.947(16)$ & $1.849(17)$ & $2.7847(12)$ & $169.0(14)$ \\
$\mathrm{N} 6-\mathrm{H} 6 C \cdots \mathrm{O} 30^{\text {vi }}$ & $0.947(16)$ & $2.590(16)$ & $3.0880(12)$ & $113.2(11)$ \\
$\mathrm{O} 31-\mathrm{H} 31 A \cdots \mathrm{O} 13^{\text {vii }}$ & $0.89(2)$ & $1.88(2)$ & $2.7418(11)$ & $165.2(17)$ \\
$\mathrm{O} 31-\mathrm{H} 31 B \cdots \mathrm{O} 11$ & $0.88(2)$ & $1.97(2)$ & $2.8184(11)$ & $162.2(16)$
\end{tabular}

Symmetry codes: (iii) $-x+1,-y+1,-z$; (iv) $x+1, y-1, z+1$; (v) $-x,-y+1,-z+1$; (vi) $x, y-1, z+1$; (vii) $-x+1,-y+1,-z+1$. 